\title{
Assessment of Psychological Comorbidities in Patients with Head and Neck Cancer as Compared to Other ENT Patients
}

\author{
Jaymin Contractor ${ }^{1}$, Varun J Dave ${ }^{2}$, Saumitra S Nemlekar ${ }^{3}$, Ravi Shah ${ }^{4}$, Ritambhara Y Mehta ${ }^{5}$, Rahul B Patel ${ }^{6}$, Jay N Suratwala ${ }^{7}$
}

\begin{abstract}
Aims and objectives:

- To determine the prevalence of psychological comorbidities in head and neck cancer (HNC) patients as compared to other ENT patients.

- To determine the number of HNC patients requiring psychiatric treatment as compared to other ENT patients.

Materials and methods: A cross-sectional study was conducted. Patients with head and neck carcinoma were selected as cases and appropriate controls were selected after matching for age ( \pm 2 years), sex, religion, address, and socioeconomic status. Both groups completed two quality of life questionnaires, Hospital Anxiety and Depression Scale (HADS), and Beck's Depression Inventory (BDI) scale. Further evaluation for depression, anxiety, or any other psychiatric illnesses was done by a psychiatrist.

Results: The cases scored significantly higher on the HADS and BDI scale and were found to have a higher incidence of depression, anxiety, suicidal tendencies, and adjustment disorders than their respective controls.

Conclusion: The prevalence of psychological comorbidities is significantly higher in head and neck carcinoma patients as compared to other ENT patients. Measuring health-related quality of life should be incorporated as an integral part of the treatment regimen and patient management. Keywords: BDI, HADS, Head and neck cancer, Psychological comorbidity, QOL in HNSCC.

Otorhinolaryngology Clinics: An International Journal (2021): 10.5005/jp-journals-10003-1373
\end{abstract}

\section{INTRODUCTION}

The head and neck carcinomas, as well as their treatment measures, cause significant dysfunction and disfigurement of the facial and pharyngolaryngeal structures. The loss of daily functions, such as swallowing, speech, smell, and taste, severely increases patient morbidity. In addition, facial disfigurement and the presence of a stoma and feeding tube among others cause drastic changes in patient's identity and personality, leading to unnecessary social intrusion and stigmata causing them to remain aloof and depressed. Thus, even if a patient is cured, the quality of life remains low. Such patients are likely to undergo some form of psychological illnesses and even suicidal tendencies along the course of the disease. Thus, it is recommended that prophylactic psychological assessment and intervention, preferably by a psychiatrist, should be performed as soon as the patient is diagnosed with carcinoma. Moreover, Health-related Quality of Life (HRQOL) should be an adjunct to the traditional end points of overall survival and disease-free survival in cancer management and be considered while formulating or choosing a treatment regimen.

\section{Materials and Methods}

The study is an observational, cross-sectional study with 50 cases and controls. Patients with histopathologically diagnosed head and neck carcinomas were selected as cases and their selection was consecutive. For each case, after matching for age ( \pm 2 years), sex, address, and socioeconomic status, a patient with a noncancerous ENT disease, e.g., goiter, cholesteatoma, vocal cord polyp, was selected as control.

Inclusion criteria: All patients giving consent and fitting in the aforementioned format were included in the study.
${ }^{1}$ Department of ENT_-Head and Neck Surgery, Government Medical College and New Civil Hospital, Surat, Gujarat, India

2,6,7 Department of Otorhinolaryngology, Government Medical College, Surat, Gujarat, India

${ }^{3-5}$ Department of Psychiatry, Government Medical College, Surat, Gujarat, India

Corresponding Author: Jaymin Contractor, Department of ENT—Head and Neck Surgery, Government Medical College and New Civil Hospital, Surat, Gujarat, India, e-mail: dr_jcontractor@yahoo.co.in

How to cite this article: Contractor J, Dave VJ, Nemlekar SS, et al. Assessment of Psychological Comorbidities in Patients with Head and Neck Cancer as Compared to Other ENT Patients. Int J Otorhinolaryngol Clin 2021;13(2):34-36.

Source of support: Nil

Conflict of interest: None

Exclusion criteria: Patients were excluded if

- They had any disease known to cause psychological abnormality.

- They had any critical illnesses (e.g., cardiorespiratory emergency).

- They were found to have a language barrier, profound hearing loss, or cognitive impairment.

- They were less than 18 years of age.

The disease, its cause, nature (benign or malignant), severity, stage, prognosis, and 5-year survival rates were then explained to the patient in full detail. These patients were then given 7 days to absorb, assess, and react to the information given to them. Then, the cases and controls underwent assessment by qualified and able psychiatrists where each patient from either group answered two questionnaires-the Hospital Anxiety and Depression Scale

() The Author(s). 2021 Open Access This article is distributed under the terms of the Creative Commons Attribution 4.0 International License (https://creativecommons. org/licenses/by-nc/4.0/), which permits unrestricted use, distribution, and non-commercial reproduction in any medium, provided you give appropriate credit to the original author(s) and the source, provide a link to the Creative Commons license, and indicate if changes were made. The Creative Commons Public Domain Dedication waiver (http://creativecommons.org/publicdomain/zero/1.0/) applies to the data made available in this article, unless otherwise stated. 
(HADS) and Beck's Depression Inventory (BDI) scale-followed by detailed psychiatric evaluation for depression, anxiety, or any other psychiatric illnesses. Data were entered into a Statistical Package for the Social Sciences format and a Chi-square test was applied to obtain statistical significance.

\section{Results}

The mean age of patients in our study was 46.4 ; of which $64 \%$ were males and $36 \%$ were females. The modified Kuppuswamy criterion was used to divide patients into different socioeconomic groups and it was found that $46 \%$ of patients belonged to lower socioeconomic strata, $44 \%$ to the middle, and $10 \%$ to the upper class. Also, $78 \%$ were Hindus and $22 \%$ were Muslims.

According to the HADS, $48 \%$ of the cases were screened positive for depression, $26 \%$ were at the borderline, and only $26 \%$ were normal as compared to controls, in which $80 \%$ were normal, $10 \%$ were at the borderline, and only $10 \%$ were screened positive for depression (Table 1). Similarly, BDI showed that $78 \%$ of cases were screened positive for depression whereas only $22 \%$ of controls were screened positive for depression (Table 2). The Chi-square test showed a highly significant association between cases with anxiety and depression as compared to controls.

After assessing patients based on the scales, they underwent personal counseling by a psychiatrist for clinical diagnosis. It was

Table 1: More number of cases presenting with depressive symptoms according to the HADS

\begin{tabular}{lccc}
\hline & \multicolumn{3}{c}{ HADS score } \\
\cline { 2 - 4 } & $0-7$ (non-case) & $8-10$ (borderline) & $>10$ (case) \\
\hline Case & $13(26 \%)$ & $13(26 \%)$ & $24(48 \%)$ \\
Control & $40(80 \%)$ & $05(10 \%)$ & $05(10 \%)$ \\
\hline
\end{tabular}

found that $62 \%$ of cases had psychiatric illnesses while only $12 \%$ of controls had psychiatric illnesses. Among the psychological comorbidities, the most common were mild-to-moderate depression (55\%) and adjustment disorders (42\%). Suicidal tendencies were found to be much more common in cases (34\%) than in controls (4\%) (Fig. 1). It was found that $78 \%$ of the cases required some forms of psychiatric intervention in the form of either psychotherapy alone or psychotherapy combined with medications, such as antidepressants, anxiolytics, etc., while only $12 \%$ of controls required such intervention (Fig. 2).

\section{Discussion}

It is evident from the study that psychological comorbidities are much more prevalent in head and neck carcinoma patients as compared to other ENT noncancerous patients. Similarly, Lydiatt et al. report that patients with HNC were at an increased risk for developing depression and anxiety, with an incidence of $15-50 \%$, as compared to patients with other forms of cancer. ${ }^{1}$ Moreover, Kam et al. proved that suicidal ideations were three times more common in HNC patients as compared to normal people. ${ }^{2}$ This is largely attributed to the disfigurement, dysfunction, and social stigma associated with $\mathrm{HNC}^{3}$ The pain, fear of recurrence, long treatment course, and the sense of impending doom play their part as well. A young, single, alcohol-dependent patient with a preexisting psychiatric illness is more likely to undergo depression and anxiety. ${ }^{4}$ The cancers of the larynx, oropharynx, and hypopharynx with a feeding tube inserted and in stage IV have been proved to be the most detrimental to patients' psychology. ${ }^{2,5,6}$

Tobacco consumption and alcoholism, the premier causes for head and neck carcinomas, have been showed to cause depression and increased suicidal tendencies too. It is well known that depression and anxiety not only affect patients' psychology but also

Table 2: BDI also suggestive of high occurrence of depression in cases as compared to controls

\begin{tabular}{lcccccc}
\hline & \multicolumn{7}{c}{ BDI score } \\
\cline { 2 - 7 } & $\begin{array}{c}\text { Normal } \\
(0-10)\end{array}$ & $\begin{array}{c}\text { Mild mood disturbance } \\
(11-16)\end{array}$ & $\begin{array}{c}\text { Borderline depression } \\
(17-20)\end{array}$ & $\begin{array}{c}\text { Moderate depression } \\
(21-30)\end{array}$ & $\begin{array}{c}\text { Severe depression } \\
(31-40)\end{array}$ & $\begin{array}{c}\text { Extreme depression } \\
(>40)\end{array}$ \\
\hline Cases & 12 & 16 & 09 & 10 & 03 & 00 \\
Control & 39 & 08 & 02 & 01 & 00 & 00 \\
\hline
\end{tabular}

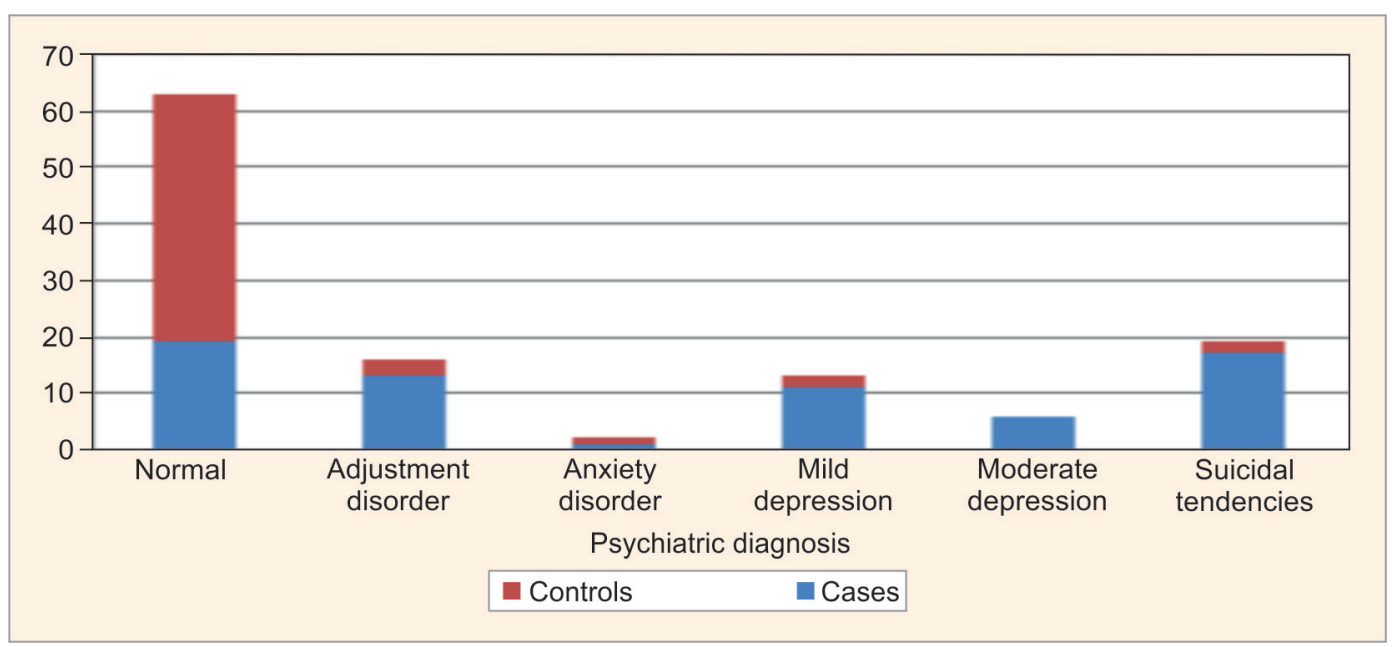

Fig. 1: High incidence of psychological comorbidities and suicidal tendencies in controls and cases 


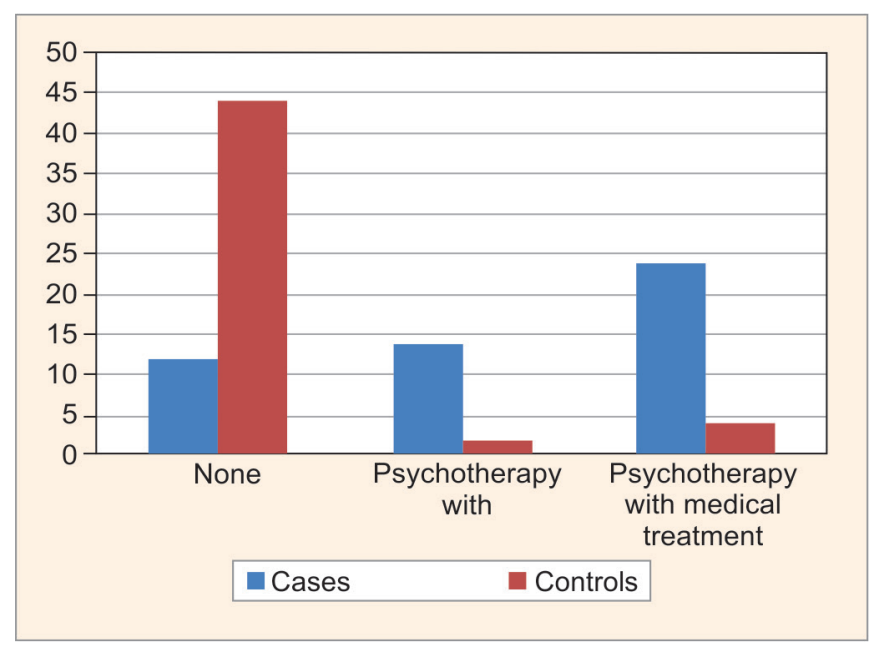

Fig. 2: Cases requiring greater psychiatric intervention as compared to controls

the general function and the persons' ability to carry out his/her routine or special activities, which further leads to nonadherence to treatment and thus increase the risk of default and recurrence. In such diseases, where it is highly likely that the patient will undergo some form of psychological illness, it is advisable that they should be protected beforehand and subjected to prophylactic psychotherapy alone or combined with medications. ${ }^{7}$

\section{Health-related Quality of Life (HRQOL)}

Today's world sees a paradigm shift as the quality of life is considered more important than quantity of life, and conventional disease end points, such as absolute survival rates and disease-free survival rates, hold little value. WHO defines "quality of life" as "an individual's perceptions of their position in life taken in the context of the culture and value systems in which they live and in relation to their goals, standards, and concerns." ${ }^{8}$ However, HRQOL is a more specific term and may be defined as "an assessment of the impact of the disease and its treatment on the physical, psychological, and social aspects of quality of life." ${ }^{\prime 9}$

Measuring HRQOL provides an insight into patients' perspective about the effects of cancer and treatment on patients' emotions, psychology, daily functions, general distress/well-being, and life as a whole. ${ }^{10}$ Moreover, given the important and subjective issue of body image after HNC, it becomes imperative to know patient perspective on it, as the surgeon is likely to misinterpret the effect of change of appearance on the patient. ${ }^{11}$ This is supported by a study conducted by Velikova et al. which suggests that routine measurement of HRQOL significantly improves patient-doctor relationship and provides emotional and mental stability, resulting in improved quality of life. ${ }^{12,13}$ More so, it is also beneficial to decide a treatment regimen when two treatments provide similar benefits and can also be used as a baseline against which the effectiveness of treatment can be measured. ${ }^{14}$ The measurement should be done preferably by a psychiatrist as studies suggest underreporting by patients in self-report questionnaires. ${ }^{15}$ Thus, measuring HRQOL will sensitize surgeons about the psychological aspect of patient care and provide holistic and complete care which will be integral in preventing psychological comorbidities and decreasing suicidal tendencies as well.

\section{Conclusion}

The prevalence of psychological comorbidities is significantly higher in head and neck carcinoma patients as compared to other ENT patients. Measurement of HRQOL should be incorporated as an integral part of treatment regimen and patient management.

\section{References}

1. Lydiatt WM, Moran J, Burke WJ. A review of depression in the head and neck cancer patient. Clin Adv Hematol Oncol 2009;7(6):397-403. Retrieved from: https://www.hematologyandoncology.net/

2. Kam D, Salib A, Gorgy G, et al. Incidence of suicide in patients with head and neck cancer. JAMA Otolaryngol Head Neck Surg 2015;141(12):1-8. DOI: 10.1001/jamaoto.2015.2480.

3. Millsopp L, Brandom L, Humphris G, et al. Facial appearance after operations for oral and oropharyngeal cancer: a comparison of casenotes and patient completed questionnaire. Br J Oral Maxillofac Surg 2006;44(5):358-363. DOI: 10.1016/j.bjoms.2005.07.017.

4. Terrell JE, Ronis DL, Fowler KE, et al. Clinical predictors of quality of life in patients with head and neck cancer. Arch Otolaryngol Head Neck Surg 2004;130(4):401-408. DOI: 10.1001/archotol.130.4.401.

5. Rogers SN. Quality of life perspectives in patients with oral cancer. Oral Oncol 2010;46(6):445-447. DOI: 10.1016/j.oraloncology. 2010.02.021.

6. Gillespie MB, Brodsky MB, Day TA, et al. Swallowing related quality of life after head and neck cancer treatment. Laryngoscope 2004;114(8):1362-1367. DOI: 10.1097/00005537-200408000-00008.

7. List MA, Stracks J, Colangelo L, et al. How do head and neck cancer patients prioritize treatment outcomes before initiating treatment? J Clin Oncol 2000;18(4):877-884. DOI: 10.1200/JCO.2000.18.4.877.

8. World Health Organization. What quality of life? The WHOQOL Group. In: World Health Forum, vol. 17. Geneva: WHO; 1996. p. 354-356.

9. Calman KC. Definitions and dimensions of quality of life. In: Aaronson NK, Beckmann J, editors. The quality of life of cancer patients. New York: Raven Press; 1987. p. 1-9.

10. Rathmell AJ, Ash DV, Howes M, et al. Assessing quality of life in patients treated for advanced head and neck cancer. Clin Oncol 1991;3(1):10-16. DOI: 10.1016/s0936-6555(05)81034-9.

11. Gliklich RE, Goldsmith TA, Funk GF. Are head and neck specific quality of life measures necessary? Head and Neck 1997;19(6):474-480. DOI: 10.1002/(sici)1097-0347(199709)19:6<474::aid-hed3>3.0.co;2-w.

12. Velikova $G$, Booth $L$, Smith $A B$, et al. Measuring quality of life in routine oncology practice improves communication and patient well-being: a randomized controlled trial. J Clin Oncol 2004;22(4):714-724. DOI: 10.1200/JCO.2004.06.078.

13. Weymuller EA, Yueh B, Deleyiannis FW, et al. Quality of life in patients with head and neck cancer: lesson learned from 549 prospectively evaluated patients. Arch Otolaryngol Head and Neck Surg 2000;126(3):329-335. DOI: 10.1001/archotol.126.3.329.

14. Rogers SN. Quality of life of head and neck cancer patients. Has treatment planning altered? Oral Oncol 2009;45(4-5):435-439. DOI: 10.1016/j.oraloncology.2008.11.006.

15. Breetvelt IS, Van Dam FS. Under reporting by cancer patients: the case of response-shift. Soc Sci Med 1991;32(9):981-987. DOI: 10.1016/02779536(91)90156-7. 